\title{
Tropical convexity via cellular resolutions
}

\author{
Florian Block · Josephine Yu
}

Received: 23 March 2005 / Accepted: 9 January 2006

(C) Springer Science + Business Media, LLC 2006

\begin{abstract}
The tropical convex hull of a finite set of points in tropical projective space has a natural structure of a cellular free resolution. Therefore, methods from computational commutative algebra can be used to compute tropical convex hulls. Tropical cyclic polytopes are also presented.
\end{abstract}

\section{Introduction}

The tropical semiring $(\mathbb{R}, \oplus, \odot)$ is the set $\mathbb{R}$ of real numbers with two binary operations called tropical addition $\oplus$ and tropical multiplication $\odot$ defined as

$$
a \oplus b=\min (a, b) \text {, and } a \odot b=a+b, \text { for all } a, b \in \mathbb{R} \text {. }
$$

Then $\mathbb{R}^{n}$ has the structure of a semimodule over the semiring $(\mathbb{R}, \oplus, \odot)$ with tropical addition

$$
\left(x_{1}, \ldots, x_{n}\right) \oplus\left(y_{1}, \ldots, y_{n}\right)=\left(x_{1} \oplus y_{1}, \ldots, x_{n} \oplus y_{n}\right),
$$

and tropical scalar multiplication

$$
c \odot\left(x_{1}, \ldots, x_{n}\right)=\left(c \odot x_{1}, \ldots, c \odot x_{n}\right) .
$$

\section{F. Block $(\bowtie)$}

Technische Universität München, Zentrum Mathematik, Boltzmannstr. 3, 85748 Garching, Germany e-mail: block@in.tum.de

J. Yu

Department of Mathematics, University of California, Berkeley, CA 94720

e-mail: jyu@math.berkeley.edu 
A set $A \subset \mathbb{R}^{n}$ is called tropically convex if for all $x, y \in A$ and $a, b \in \mathbb{R}$ also $(a \odot$ $x) \oplus(b \odot y) \in A$. Notice that we do not put any extra condition on $a$ and $b$ as in usual convexity. The tropical convex hull tconv $(V)$ of a set $V \subset \mathbb{R}^{n}$ is the inclusionwise minimal, tropically convex set containing $V$ in $\mathbb{R}^{n}$. Also,

$$
\operatorname{tconv}(V)=\left\{\left(a_{1} \odot v_{1}\right) \oplus \cdots \oplus\left(a_{r} \odot v_{r}\right): v_{1}, \ldots, v_{r} \in V \text { and } a_{1}, \ldots, a_{r} \in \mathbb{R}\right\}
$$

Since any tropically convex set $A$ is closed under tropical scalar multiplication, we identify it with its image under the projection onto the $(n-1)$-dimensional tropical projective space

$$
\mathbb{T} \mathbb{P}^{n-1}=\mathbb{R}^{n} /(1, \ldots, 1) \mathbb{R}
$$

The tropical convex hull of a finite set of points has a natural structure of a polyhedral complex. We refer to [2] for a more extensive introduction to tropical convexity.

Let $V=\left\{v_{1}, \ldots, v_{r}\right\} \subset \mathbb{T P}^{n-1}, v_{i}=\left(v_{i 1}, \ldots, v_{i n}\right)$, and $\mathcal{P}=\operatorname{tconv}(V)$. Let $S=$ $\mathbb{R}\left[x_{11}, \ldots, x_{r n}\right]$ be the polynomial ring over $\mathbb{R}$ with indeterminates $x_{i j}$ for $i \in[r]=$ $\{1, \ldots, r\}$ and $j \in[n]=\{1, \ldots, n\}$. Let the weight of $x_{i j}$ be $v_{i j}$ and the weight of a monomial $\mathbf{x}^{\mathbf{a}}=\prod x_{i j}^{a_{i j}} \in S$ be $\sum a_{i j} v_{i j}$. The initial form $\operatorname{in}_{V}(f)$ of a polynomial $f=$ $\sum c_{i} \mathbf{x}^{\mathbf{a}_{i}}$ is defined to be the sum of terms $c_{i} \mathbf{x}^{\mathbf{a}_{i}}$ such that $\mathbf{x}^{\mathbf{a}_{i}}$ has maximal weight. Let $J$ be the ideal generated by the $2 \times 2$ minors of the $r \times n$ matrix $\left[x_{i j}\right]$. Let $I=\operatorname{in}_{V}(J)=$ $\left\langle\operatorname{in}_{V}(f): f \in J\right\rangle$ be the initial ideal of $J$ with respect to $V$. If $V$ is sufficiently generic, the initial ideal $I$ is a square free monomial ideal. The square free Alexander dual $I^{*}$ of a square free monomial ideal $I=\left\langle\mathbf{x}^{\mathbf{a}^{1}}, \ldots, \mathbf{x}^{\mathbf{a}^{k}}\right\rangle$ is

$$
I^{*}=m^{\mathbf{a}^{1}} \cap \cdots \cap m^{\mathbf{a}^{k}},
$$

where each $\mathbf{a}^{i}$ is a $0-1$ vector and $m^{\mathbf{a}}=\left\langle x_{j}: a_{j}=1\right\rangle$. See $[6,11]$ for details. The following is our main result.

Theorem 1. For a sufficiently generic set of points $V$ in $\mathbb{T} \mathbb{P}^{n-1}$, the tropical convex hull $\mathcal{P}=\operatorname{tconv}(V)$ supports a minimal linear free resolution of the ideal $I^{*}$, as a cellular complex.

Moreover, the cellular structure of the minimal free resolution is unique (Remark 7), so we get the following algorithm for computing the tropical convex hull of a finite set of points in tropical projective space.

\section{Algorithm 2.}

Input $A$ list of points $v_{1}, \ldots, v_{r} \in \mathbb{T} \mathbb{P}^{n-1}$ in generic position.

Output The tropical convex hull of the input points.

\section{Algorithm}

1. Set $J=\left\langle 2 \times 2\right.$ minors of the $r \times n$ matrix $\left.\left[x_{i j}\right]\right\rangle$.

2. Compute $I=\operatorname{in}_{V}(J)$.

3. Compute the Alexander dual $I^{*}$ of $I$. 
Fig. 1 Tropical convex hull of four points in $\mathbb{T P}^{2}$.

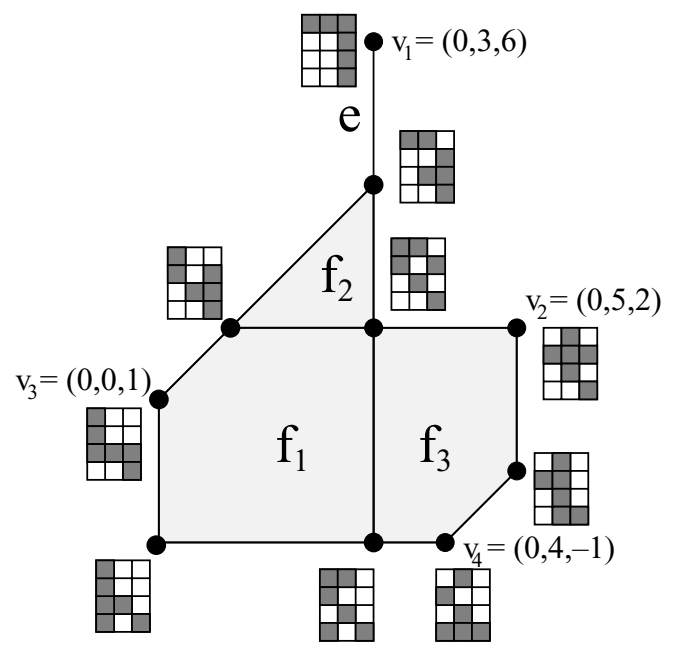

4. Find a minimal free resolution of $I^{*}$.

5. Output the desired data about the tropical polytope.

A typical output for $r=4$ and $n=3$ is depicted in Figure 1. The ten grids represent ten square free monomials in $S$ of degree six, where each unshaded box represents an indeterminate in the monomial. The cell complex is the minimal free resolution of their ideal.

Since the set of $2 \times 2$ minors of a matrix is fixed under transposition of the matrix, we immediately see the duality between tropical convex hulls of $r$ points in $\mathbb{T P}^{n-1}$ and $n$ points in $\mathbb{T} \mathbb{P}^{r-1}$, as shown in [2].

The rest of this paper is organized as follows: In Section 2 we prove Theorem 1 and demonstrate the algorithm with an example. In Section 3 we deal with algorithmic and computational aspects. We suggest ways to deal with non-generic points and to get an exterior (halfspace) description of a tropical polytope. We also discuss the efficiency of Algorithm 2. Finally, we study tropical cyclic polytopes in Section 4.

\section{From geometry to algebra and back}

We first describe the polyhedral complex structure of the tropical polytope $\mathcal{P}$. Let $W=\mathbb{R}^{r+n} /(1, \ldots, 1,-1, \ldots,-1) \mathbb{R}$. Define an unbounded polyhedron as follows:

$$
\mathcal{P}_{V}=\left\{(y, z) \in W: y_{i}+z_{j} \leq v_{i j} \text { for all } i \in[r], j \in[n]\right\} .
$$

By [2], there is a piecewise linear isomorphism between the complex of bounded faces of $\mathcal{P}_{V}$ and the tropical polytope $\mathcal{P}=\operatorname{tconv}(V)$ given by the projection $(y, z) \mapsto z$. The boundary complex $\partial \mathcal{P}_{V}$ of $\mathcal{P}_{V}$ is polar to the regular polyhedral subdivision of the product of simplices $\Delta_{r-1} \times \Delta_{n-1}$ induced by the weights $v_{i j}$. We denote this regular subdivision by $\left(\partial \mathcal{P}_{V}\right)^{*}$. More precisely, a subset of vertices $\left(e_{i}, e_{j}\right)$ of $\Delta_{r-1} \times \Delta_{n-1}$ 
forms a cell of the subdivision $\left(\partial \mathcal{P}_{V}\right)^{*}$ if and only if the equations $y_{i}+z_{j}=v_{i j}$ indexed by these vertices specify a face of the polyhedron $\mathcal{P}_{V}$.

Let $\mathcal{A}$ denote the $(r+n) \times r n$ integer matrix whose column vectors are the vertices $\left(e_{i}, e_{j}\right)$ of $\Delta_{r-1} \times \Delta_{n-1}$, where $i \in[r], j \in[n]$. This defines a homomorphism $\mathbb{Z}^{r n} \rightarrow$ $\mathbb{Z}^{r+n}$ by $e_{i j} \mapsto\left(e_{i}, e_{j}\right)$. Let $L$ denote its kernel. The ideal $J$ generated by the $2 \times 2$ minors of $\left[x_{i j}\right]$ is the (toric) lattice ideal

$$
J=\left\langle\mathbf{x}^{\mathbf{a}}-\mathbf{x}^{\mathbf{b}}: \mathbf{a}, \mathbf{b} \in \mathbb{N}^{r n} \text { with } \mathbf{a}-\mathbf{b} \in L\right\rangle .
$$

See [6, Chapter 7] or [11, Chapter 8] for details about lattice ideals.

Lemma 3. The initial ideal I is independent of the representatives of the points $v_{i}$ in the tropical projective space. In other words, if $c \cdot(1,1, \ldots, 1)$ is added to any $v_{i}$, the initial ideal I remains the same.

Proof: The ideal $J$ is homogeneous with respect to any grading assigning the same weight to the variables in each row.

In the rest of this section, we will assume that the points $v_{1}, \ldots, v_{r}$ are in generic position, i.e., they satisfy the conditions in the next result.

Proposition 4. The following are equivalent.

(1) The initial ideal I is a monomial ideal.

(2) The regular subdivision $\left(\partial P_{V}\right)^{*}$ of $\Delta_{r-1} \times \Delta_{n-1}$ induced by the weights $v_{i j}$ is a triangulation.

(3) The polyhedron $\mathcal{P}_{V}$ is simple.

(4) For any $k$ distinct points in $V$, their projections onto a $k$-dimensional coordinate subspace do not lie in a tropical hyperplane, for any $2 \leq k \leq n$.

(5) No $k \times k$ submatrix of the $r \times n$ matrix $\left[v_{i j}\right]$ is tropically singular, i.e., has vanishing tropical determinant (e.g. see [2]), for any $2 \leq k \leq n$.

Proof: $(2) \Longleftrightarrow$ (3) follows directly from the polarity between the regular subdivisions of $\overline{\Delta_{r-1} \times \Delta_{n-1}}$ and $\partial \mathcal{P}_{V}$.

(2) $\Longleftrightarrow(5)$ is proven in [2, Proposition 24].

(4) $\Longleftrightarrow(5)$ is proven in [9, Lemma 5.1].

$(1) \Longleftrightarrow(2)$ : Statement (1) is equivalent to $V$ being in the interior of a full dimensional cone in the Gröbner fan of the lattice ideal $J$. Statement (2) means that $V$ is in the interior of a full dimenional cone in the secondary fan $\mathcal{N}(\Sigma(\mathcal{A})$ ) which is the normal fan of the secondary polytope of $\mathcal{A}$ (for details see [11]). By [11, Proposition 8.15(a)], these two fans coincide if $\mathcal{A}$ is unimodular, i.e., all invertible $\operatorname{rank}(\mathcal{A}) \times \operatorname{rank}(\mathcal{A})$ submatrices have the same determinant up to sign. We will check criterion (iv) of [10, Theorem 19.3] for total unimodularity. Fix a collection of rows of $\mathcal{A}$. Split it according to containment in the upper $r \times r n$ submatrix of the $(r+n) \times r n$ matrix $\mathcal{A}$. Then the sum of the rows in each part is a $0-1$ vector. This implies that all submatrices of $\mathcal{A}$ have determinants 0 or \pm 1 , so $\mathcal{A}$ is unimodular. 
It also follows from the unimodularity that all monomial initial ideals of $J$ are square free [11, Corollary 8.9]. Let $\Delta_{V}(J)$ be the initial complex of $J$, i.e., the simplicial complex whose Stanley-Reisner ideal (see $[6,11])$ is $I=\operatorname{in}_{V}(J)$. We can identify a square free monomial $m \in S$ with the set of indeterminates $x_{i j}$ dividing $m$. The vertices of $\Delta_{V}(J)$ are $x_{i j}$, and the minimal generators of $I$ are the minimal non-faces of $\Delta_{V}(J)$. Moreover, the minimal generators of the Alexander dual $I^{*}$ are the complements of the maximal cells of $\Delta_{V}(J)$. The following lemma follows immediately from [6, Theorem 7.33] or [11, Theorem 8.3] and establishes a connection between the ideal $J$ and the tropical convex hull.

Lemma 5. We have an isomorphism $\Delta_{V}(J) \cong\left(\partial \mathcal{P}_{V}\right)^{*}$, as cell complexes. In particular, there is a bijection between maximal cells of $\Delta_{V}(J)$ and those of $\left(\partial \mathcal{P}_{V}\right)^{*}$ induced by $x_{i j} \longleftrightarrow\left(e_{i}, e_{j}\right)$.

We will label the vertices of $\mathcal{P}_{V}$ by the minimal generators of $I^{*}$ so that $\mathcal{P}_{V}$ gives a cellular resolution of $I^{*}$. First, we have a general lemma about simple polyhedra which can be proved using [6, Proposition 4.5].

Lemma 6 (Section 4.3.6 and Exercises 4.5-6). Let $P$ be a simple polyhedron (possibly unbounded) with facets $F_{1}, \ldots, F_{m}$. Label each face $G$ of $P$ by $\mathbf{x}^{\mathbf{a}_{G}}=\prod_{F_{i} \nsupseteq G} x_{i} \in$ $\mathbb{R}\left[x_{1}, \ldots, x_{m}\right]$. Then the complex of bounded faces of $P$ supports a minimal linear free resolution of the square free monomial ideal generated by the vertex labels.

We will apply this to $\mathcal{P}_{V}$ to prove Theorem 1 stated in the introduction.

Proof of Theorem 1: Since $V$ is generic, $\mathcal{P}_{V}$ is simple. Hence, by Lemma 6, the tropical convex hull $\mathcal{P}$, which is isomorphic to the complex of bounded faces of $\mathcal{P}_{V}$, supports a minimal linear free resolution of the ideal generated by the monomial labels of its vertices. We only need to show that the labels from Lemma 6 coincide with the minimal generators of $I^{*}$.

The facets $F_{i j}$ of $\mathcal{P}_{V}$ are defined by equations $y_{i}+z_{j}=v_{i j}$. Let $x_{i j}$ be the indeterminate corresponding to $F_{i j}$. For a square free monomial $m$,

$m$ is a vertex label of $\mathcal{P}_{V} \Longleftrightarrow \bigcap\left\{F_{i j}: x_{i j}\right.$ does not divide $\left.m\right\}$ is a vertex of $\mathcal{P}_{V}$

$\Longleftrightarrow\left\{\left(e_{i}, e_{j}\right): x_{i j}\right.$ does not divide $\left.m\right\}$ is a maximal cell of $\left(\partial \mathcal{P}_{V}\right)^{*}$

$\Longleftrightarrow\left\{x_{i j}: x_{i j}\right.$ does not divide $\left.m\right\}$ is a maximal cell of $\Delta_{V}(J)$

$\Longleftrightarrow$ is a minimal generator of $I^{*}$.

The third equivalence follows from Lemma 5.

Remark 7. By construction, the monomial labels are unique, so all the multi-graded Betti numbers are at most one. This combined with the linearity of the resolution implies that the cellular structure of the minimal free resolution is unique.

However, the multi-graded Betti numbers already determine the tropical polytope because in this case a face $F$ contains a face $G$ if and only if the monomial label of 
Fig. 2 Grids representing

$x_{21} x_{22} x_{31} x_{32} x_{41} x_{42}$ and

$x_{11} x_{13} x_{31} x_{33} x_{41} x_{42}$ for

$r=4, n=3$. These are the

labels of $v_{1}$ and $v_{2}$ in Figure 1.
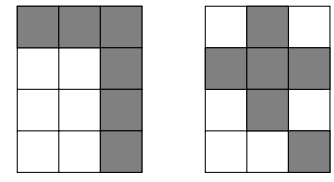

$F$ is divisible by the monomial label of $G$. Moreover, the vertex labels (the minimal generators of $I^{*}$ ) determine all the other monomial labels by Lemma 12 .

The dimension $\operatorname{dim}(U)$ of any subset $U$ of $\mathbb{T} \mathbb{P}^{n-1}$ is the affine dimension of its projection $\left\{u \in \mathbb{R}^{n}:(0, u) \in U\right\}$ onto the last $n-1$ coordinates.

Corollary 8. For any face $F \subset \mathcal{P}, \operatorname{dim}(F)=\operatorname{deg}\left(\mathbf{x}^{\mathbf{a}_{F}}\right)-(n-1)(r-1)$.

The monomial labels have a geometric meaning. To have a more intuitive notation, we will represent each square free monomial $m \in S$ with an $r \times n$ grid shaded at position $(i, j)$ if $x_{i j}$ does not divide $m$. Hence the support of $\mathbf{x}^{\mathbf{a}_{F}}$ is left unshaded in the grid (see Figure 2). Let $C_{j}=$ cone $\left\{e_{i}: i \neq j\right\}$ be the closed cone which is the usual conical (positive) hull of all but one standard unit vector. Suppose $z=\left(z_{1}, \ldots, z_{n}\right) \in \mathcal{P}$ is in the relative interior of a cell with label $\mathbf{x}^{\mathbf{a}_{z}}$, and it is the image of the point $(y, z) \in \mathcal{P}_{V}$. Then

$$
\begin{aligned}
& x_{i j} \backslash \mathbf{x}^{\mathbf{a}_{z}} \Longleftrightarrow\left\{\begin{array}{c}
y_{i}+z_{j}=v_{i j} \\
y_{i}+z_{k} \leq v_{i k}
\end{array} \forall k\right\} \Longleftrightarrow v_{i j}-z_{j} \leq v_{i k}-z_{k} \quad \forall k \\
& \Longleftrightarrow v_{i}-z \in C_{j} \Longleftrightarrow v_{i} \in z+C_{j} \text {. }
\end{aligned}
$$

So the box $(i, j)$ is shaded if and only if the input vertex $v_{i}$ lies in the sector $z+C_{j}$. See Figure 3(b).

This monomial labeling is essentially the same as the labeling by types introduced in [2]. Specifically, for any point $z$ in the relative interior of a cell $F$ in $\mathcal{P}$ with type $(z)=$ $\left(S_{1}, \ldots, S_{n}\right)$, we have $i \in S_{j}$ if and only if $x_{i j}$ does not divide $\mathbf{x}^{\mathbf{a}_{F}}$. The following result follows from [2, Lemma 10].

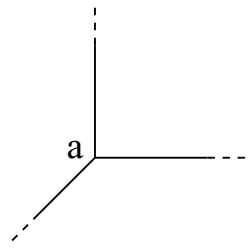

(a)

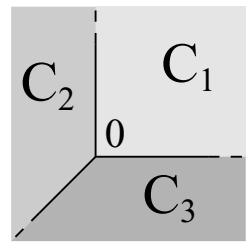

(b)

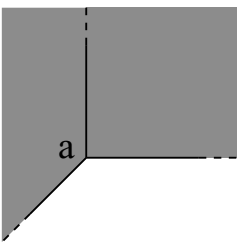

(c)

Fig. 3 (a) Tropical hyperplane in $\mathbb{T} \mathbb{P}^{2}$ with apex $a$. (b) The sectors at apex 0 in $T \mathbb{P}^{2}$. (c) Tropical halfspace $(a,\{1,2\})$ in $\mathbb{T P}^{2}$. 
Lemma 9. Given the monomial label $\mathbf{x}^{\mathbf{a}_{z}}$ of a vertex $z$, its coordinates can be computed by solving the linear system

$$
\left\{z_{l}-z_{k}=v_{i l}-v_{i k}: i \in[r], k, l \in[n], x_{i k} \text { and } x_{i l} \text { do not divide } \mathbf{x}^{\mathbf{a}_{z}}\right\} .
$$

Example 10. (Four Points in $\mathbb{T} \mathbb{P}^{2}$.) Assume we are given the following points in $\mathbb{T P}^{2}$ $(r=4, n=3)$ :

$$
v_{1}=(0,3,6), v_{2}=(0,5,2), v_{3}=(0,0,1), v_{4}=(0,4,-1) .
$$

They determine the tropical polytope in Figure 1. The points give the weight vector $V=(0,3,6,0,5,2,0,0,1,0,4,-1)$ in the polynomial ring $S=\mathbb{R}\left[x_{11}, x_{12}\right.$, $\left.x_{13}, x_{21}, x_{22}, x_{23}, x_{31}, x_{32}, x_{33}, x_{41}, x_{42}, x_{43}\right]$. The initial ideal $I$ and its Alexander dual are

$$
\begin{aligned}
I= & \operatorname{in}_{V}\left\langle 2 \times 2 \text { minors of }\left[\begin{array}{lll}
x_{11} & x_{12} & x_{13} \\
x_{21} & x_{22} & x_{23} \\
x_{31} & x_{32} & x_{33} \\
x_{41} & x_{42} & x_{43}
\end{array}\right]\right\rangle \\
= & \left\langle x_{33} x_{41}, x_{23} x_{41}, x_{23} x_{31}, x_{12} x_{31}, x_{31} x_{11} x_{42}, x_{11} x_{42}, x_{31} x_{41}, x_{31} x_{31}, x_{13} x_{21},\right. \\
& \left.x_{33} x_{42}, x_{22} x_{41}, x_{13} x_{32}, x_{22} x_{31}, x_{11} x_{22}, x_{23} x_{42}, x_{22} x_{33}, x_{13} x_{42}, x_{13} x_{22}, x_{12} x_{21} x_{33}\right\rangle, \\
I^{*}= & \left\langle x_{12} x_{13} x_{22} x_{23} x_{33} x_{42}, x_{12} x_{13} x_{22} x_{23} x_{41} x_{42}, x_{13} x_{22} x_{23} x_{31} x_{33} x_{42}, x_{12} x_{13} x_{22} x_{31} x_{41} x_{42},\right. \\
& x_{13} x_{22} x_{31} x_{33} x_{41} x_{42}, x_{13} x_{21} x_{22} x_{31} x_{41} x_{42}, x_{11} x_{13} x_{22} x_{23} x_{31} x_{33}, x_{21} x_{22} x_{31} x_{32} x_{41} x_{42}, \\
& \left.x_{11} x_{13} x_{31} x_{33} x_{41} x_{42}, x_{11} x_{13} x_{23} x_{31} x_{33} x_{41}\right\rangle .
\end{aligned}
$$

Note that $I$ is not generated in degree 2. Compare the minimal generators of $I^{*}$ with the grids in Figure 1. The minimal free resolution of $I^{*}$ is of the form

$$
0 \longleftarrow I^{*} \stackrel{M_{0}}{\longleftarrow} S^{10} \stackrel{M_{1}}{\longleftarrow} S^{12} \stackrel{M_{2}}{\longleftarrow} S^{3} \longleftarrow 0 .
$$

The tropical convex hull consists of 10 zero-dimensional faces (vertices), 12 onedimensional faces (edges), and 3 two-dimensional faces.

Table 1 shows the monomial matrix $M_{2}$ in the monomial matrix notation of [6, Section 1.4]. The rows correspond to the edges of the tropical polytope, and the three columns, whose labels are omitted here, correspond to the faces $f_{1}, f_{2}$, and $f_{3}$, respectively.

\section{Algorithmic and computational aspects}

Let $0 \leftarrow I^{*} \leftarrow F_{0} \leftarrow \cdots \leftarrow F_{m}$ be the free resolution computed by the algorithm, and let $M_{i}: F_{i} \rightarrow F_{i-1}$ denote the monomial matrices defining the boundary maps. Since the free resolution is linear, the row labels of the matrix $M_{i}$ are in one-to-one 
Table 1 Monomial matrix $M_{2}$ in Example 10.

$\begin{array}{lllllll}x_{12} & x_{13} & x_{22} & x_{23} & x_{31} & x_{33} & x_{42} \\ x_{12} & x_{13} & x_{22} & x_{23} & x_{33} & x_{41} & x_{42} \\ x_{12} & x_{13} & x_{22} & x_{23} & x_{31} & x_{41} & x_{42} \\ x_{12} & x_{13} & x_{22} & x_{31} & x_{33} & x_{41} & x_{42} \\ x_{11} & x_{13} & x_{22} & x_{23} & x_{31} & x_{33} & x_{42} \\ x_{12} & x_{13} & x_{21} & x_{22} & x_{31} & x_{41} & x_{42} \\ x_{13} & x_{22} & x_{23} & x_{31} & x_{33} & x_{41} & x_{42} \\ x_{13} & x_{21} & x_{22} & x_{31} & x_{32} & x_{41} & x_{42} \\ x_{11} & x_{13} & x_{22} & x_{31} & x_{33} & x_{41} & x_{42} \\ x_{13} & x_{21} & x_{22} & x_{31} & x_{33} & x_{41} & x_{42} \\ x_{11} & x_{13} & x_{22} & x_{23} & x_{31} & x_{33} & x_{41} \\ x_{11} & x_{13} & x_{23} & x_{31} & x_{33} & x_{41} & x_{42}\end{array} \quad\left[\begin{array}{ccc}1 & 0 & 0 \\ -1 & 0 & 0 \\ -1 & 0 & 0 \\ -1 & 1 & 0 \\ 0 & 0 & -1 \\ 0 & -1 & 0 \\ 1 & 0 & -1 \\ \mathbf{0} & \mathbf{0} & \mathbf{0} \\ 0 & 0 & 1 \\ 0 & 1 & 0 \\ 0 & 0 & -1 \\ 0 & 0 & 1\end{array}\right]$

correspondence with the faces of dimension $i-1$, its column labels with the faces of dimension $i$. An entry in $M_{i}$ is nonzero if and only if its row label divides its column label, which happens if and only if the face corresponding to its column contains the face corrresponding to its row. Therefore the number of $i$-dimensional faces with $k$ facets in the tropical convex hull is equal to the number of columns of $M_{i}$ having $k$ nonzero entries. A face $F$ is maximal if and only if it has dimension $n-1$ or the row in $M_{\operatorname{dim}(F)+1}$ labeled by $\mathbf{x}^{\mathbf{a}_{F}}$ contains zeroes only. So the eighth row in Table 1 corresponds to edge $e$ in Figure 1, which is not contained in any other face.

We can also compute the $f$-matrix $\left[f_{i j}\right](0 \leq i \leq n-1,1 \leq j)$ where $f_{i j}$ is the number of faces having dimension $i$ and $j$ vertices. We already know the $f$-vector $\sum_{j} f_{i j}$ which is the sum of columns in the $f$-matrix. The following result in [2] was obtained by counting regular triangulations of $\Delta_{r-1} \times \Delta_{n-1}$.

Proposition 11 (2, Corollary 25). All tropical convex hulls of $r$ generic points in $T \mathbb{P}^{n-1}$ have the same $f$-vector. The number of faces of dimension $i$ is equal to the multinomial coefficient

$$
\left(\begin{array}{c}
r+n-i-2 \\
r-i-1, n-i-1, i
\end{array}\right)=\frac{(r+n-i-2) !}{(r-i-1) ! \cdot(n-i-1) ! \cdot i !} .
$$

A combinatorial algorithm for building the face poset

Given the vertex labels of $\mathcal{P}$, we can compute the whole face poset of $\mathcal{P}$ combinatorially. The following result follows from [2, Corollary 14] and Corollary 8.

Lemma 12. Let $F$ be a face of $\mathcal{P}$ with grid $a_{F}$ and let $b$ be a grid arising from $a_{F}$ by unshading one box such that no row or column is completely unshaded. Then there is a face $G \supset F$ with label $a_{G}=b$ and of one dimenstion higher.

Conversely, every face can be obtained this way starting from the vertices. So, instead of computing the free resolution, we can build the face poset combinatorially if we know the vertex labels, i.e., the minimal generators of $I^{*}$. We have an implementation of this algorithm using Macaulay 2 [3], Maple [5], and JavaView [8]. 
Table 2 Computation times (in seconds) for $I, I^{*}$, and the free resolution for tropical cyclic polytopes $C_{r, n}$.

\begin{tabular}{ccccc}
\hline $\mathrm{n}$ & $\mathrm{r}$ & Initial ideal & Alexander dual & Free resolution \\
\hline 3 & 30 & 74 & 433 & 2 \\
4 & 21 & 64 & 944 & 23 \\
6 & 10 & 15 & 221 & 27 \\
8 & 10 & 70 & 4169 & 1106 \\
\hline
\end{tabular}

Remark 13 (Non-generic Input Vertices). When the input vertices $V$ are not in generic position, the initial ideal $I$ is not monomial. In that case, we can replace the weights $V$ with any refinement which makes $I$ a monomial ideal and proceed as before to build the face poset. We can then compute the coordinates of the vertices using Lemma 9 and identify vertices with the same coordinates. We suggest this way without having a proof.

\section{Tropical halfspaces}

Tropical halfspaces introduced in [4] give us an exterior description of tropical polytopes. We can extend our algorithm to find such a description.

The tropical hyperplane at the apex $a \in \mathbb{T P}^{n-1}$ is the set which is the union of boundaries of the sectors $a+C_{i}$ (see Figure 3). For $a \in \mathbb{T P}^{n-1}, \emptyset \neq A \subsetneq[n]$, the set $a+\bigcup_{i \in A} C_{i}$ is a closed tropical halfspace (see Figure 3(c)). Tropical halfspaces are tropically convex, and a tropical polytope $\mathcal{P}$ is the intersection of the inclusionwise minimal halfspaces containing it [4]. The apex of such a minimal halfspace must be a vertex of $\mathcal{P}$ on the boundary [4, Lemma 3.6]. Recall that the box $(i, j)$ in the grid label of a vertex $v$ is shaded if and only if $v_{i} \in v+C_{j}$. Hence $\mathcal{P}$ is the intersection of the halfspaces $v+\bigcup_{i \in A} C_{i}$ such that $v$ is a vertex of $\mathcal{P}$ and $A$ is a minimal subset of columns in the corresponding grid of $v$ such that the shaded boxes in those column cover all the rows. This description is redundant in general. We may be able to refine this result as follows.

Conjecture 14. In the generic case, a minimal halfspace with respect to $\mathcal{P}$ has the form $v+\bigcup_{i \in A} C_{i}$ where $v$ is a vertex of $\mathcal{P}$ and in the grid label of $v$ the shaded boxes in the columns in $A$ form a partition of $[r]$.

The converse of the conjecture above is not true, i.e., there are non-minimal halfspaces of the form described.

Experiments with computation time

We experimented with computing tropical cyclic polytopes $C_{r, n}$ (which will be defined in the next section) with $r$ input vertices in $n-1$ (projective) dimensions. We used Macaulay 2 [3] on a Sun Blade 150 (UltraSPARC-IIe $550 \mathrm{MHz}$ ) computer with 512 MB memory. The computation became infeasible when $r n>80$ or so, although $r=$ $30, n=3$ worked. The main problem was the insufficient amount of memory. Some sample computation times for tropical cyclic polytopes are given in Table 2 . We see from the data that computing the Alexander dual can be a problem. This can be made faster using the Monos Language for Monomial Decompositions [7]. 


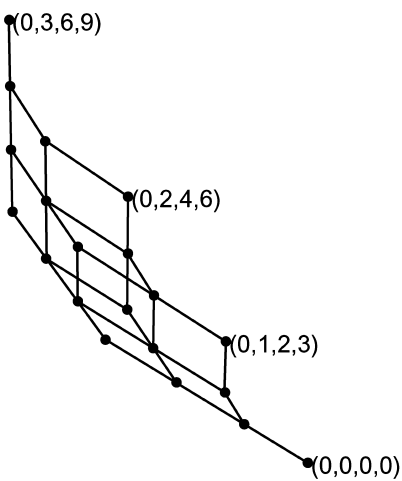

(a)

\begin{tabular}{|c|c|c|c|}
\hline $\mathrm{X}_{11}$ & $\mathrm{X}_{12}$ & $\ldots$ & 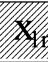 \\
\hline$X_{21}$ & 2 & G & 2 \\
\hline$\vdots$ & III & $\because$ & ! \\
\hline-1 & -1 & $\ldots$ & \\
\hline
\end{tabular}

(b)

Fig. 4 (a) Tropical cyclic polytope on four vertices in $\mathbb{T P}^{3}$. (b) A path corresponding to a generator of the Alexander dual $I^{*}$.

\section{Tropical cyclic polytopes}

Define tropical cyclic polytopes as $C_{r, n}=\operatorname{tconv}\left\{v_{1}, \ldots, v_{r}\right\} \subset \mathbb{T} \mathbb{P}^{n-1}$, where $v_{i j}=$ $(i-1)(j-1)$ for $i \in[r], \quad j \in[n]$. Since $(i-1)^{\odot(j-1)}=(i-1)(j-1)$, this is tropical exponentiation. The $C_{r, n}$ are generic because the minimum in any $k \times k$ minor of the matrix $\left[v_{i j}\right]$ is attained uniquely by the antidiagonal. An example of a tropical cyclic polytope is shown in Figure 4(a).

The $2 \times 2$ minors of $\left[x_{i j}\right]$ form a Gröbner basis with respect to $V$, and the initial ideal $I$ is the diagonal initial ideal generated by the binomials which are on the diagonals of the $2 \times 2$ minors. This correspond to the staircase triangulation of $\Delta_{r-1} \times \Delta_{n-1}$.

Consider a path in an $r \times n$ grid representing indeterminates $x_{i j}$, which goes from the lower left corner to the upper right corner, only moving either right or up at each step as in Figure 4(b). Such paths are precisely the maximal sets, with respect to inclusion, that do not contain diagonal pairs. Hence their complements correspond to the minimal generators of the Alexander dual $I^{*}$, which are the monomial labels of the vertices of $C_{r, n}$.

The labels of the faces of the tropical cyclic polytope $C_{r, n}$ are obtained by unshading the boxes on the paths so that the remaining shaded set still intersects every row and
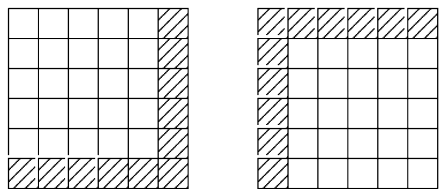

(a)

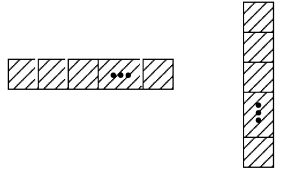

(b)

Fig. 5 (a) Paths in grids corresponding to two 1-valent vertices in $C_{r, n}$. (b) Horizontal and vertical stripes. 
Fig. 6 (a) A diagonal step. (b) Corners indicating that the corresponding monomials are not minimal.

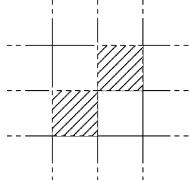

(a)

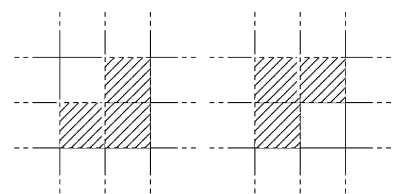

(b)

every column. For example, there are two 1-valent vertices with grids corresponding to the paths in Figure 5(a). The two edges containing these vertices are the only maximal 1 -faces, whose labels are obtained by unshading the lower right corner and the upper left corner, respectively.

We can identify a vertex of $C_{r, n}$ with the Young diagram above (or below) the corresponding path in the $r \times n$ grid. Then the 1-skeleton of $C_{r, n}$ is the Hasse diagram of the Young lattice of the Young diagram fitting in an $(r-1) \times(n-1)$ grid.

The shaded part in the label of a $k$-dimensional face contains the diagonal steps as in Figure 6(a) exactly $k$ times because every time we shade in such a corner, the dimension decreases by one. By straightforward counting, we get that

$$
\# k \text {-faces in } C_{r, n}=\left(\begin{array}{c}
r+n-k-2 \\
r-k-1, n-k-1, k
\end{array}\right)
$$

as seen in Proposition 11. That is, out of the $r+n-k-2$ steps we take from the lower left corner to the upper right corner, we take $r-k-1$ steps up, $n-k-1$ steps right, and $k$ steps diagonally.

Proposition 15. The exponential generating function for the numbers $M_{r, n, k}$ of maximal $k$-faces of the tropical cyclic polytope $C_{r, n}$ is

$$
\sum_{r \geq 1, n \geq 1, k \geq 0} \frac{M_{r, n, k}}{r ! n ! k !} x^{r} y^{n} z^{k}=\frac{\partial}{\partial z} \exp \left(z\left(y e^{x}-y+x e^{y}-x-x y\right)\right),
$$

and the ordinary generating function is

$$
\sum_{r \geq 1, n \geq 1, k \geq 0} M_{r, n, k} x^{r} y^{n} z^{k}=\left(\frac{x y}{1-y}+\frac{y x^{2}}{1-x}\right) /\left(1-z\left(\frac{x y}{1-y}+\frac{y x^{2}}{1-x}\right)\right) .
$$

Proof: A face is maximal if and only if the set of shaded boxes in the $r \times n$ grid does not contain any corners as in Figure 6(b). Then $M_{r, n, k}$ is equal to the number of $(k+1)$-tuples of either horizontal or vertical stripes of boxes, as in Figure 5(b), such that the sum of the widths equals $n$ and the sum of heights equals $r$. The proposition follows from basic properties of generating functions.

Moreover, every $k$-dimensional face contains precisely $2^{k}$ vertices because every diagonal step as in Figure 6(a) gives 2 ways of shading in the corners, and there are exactly $k$ such diagonal steps. From this it is easy to see that every $k$-dimensional face 
has the combinatorial structure of a $k$-dimensional hypercube. Therefore, the $f$-matrix of $C_{r, n}$ is very simple: $f_{k, 2^{k}}=\left(\begin{array}{c}r+n-k-2 \\ r-k-1, n-k-1, k\end{array}\right)$, and all other entries are 0 .

\section{Conclusion and future directions}

The methods we described can be applied to a wide range of combinatorial objects which are dual to triangulations of polytopes. For example, tight spans of finite metric spaces can be computed using the $2 \times 2$ minors of a symmetric matrix. There are also many enumerative questions about tropical polytopes. For example, very little is known about the $f$-matrices.

Acknowledgements This paper grew out of a term project from the course "Combinatorial Commuative Algebra" taught by Bernd Sturmfels at UC Berkeley in the fall semester 2004. We are very grateful to Bernd Sturmfels for all his guidance, stimulating discussions, and inspiring questions. We also thank Mike Develin, Hiroshi Hirai, Michael Joswig, David Speyer, and the referees for helpful comments and suggestions. Florian Block held a DAAD scholarship, and Josephine Yu was supported by an NSF Graduate Research Fellowship.

\section{References}

1. D. Eisenbud, Commutative Algebra with a View toward Algebraic Geometry, Graduate Texts in Mathematics, Springer, 1995.

2. M. Develin and B. Sturmfels, "Tropical Convexity", Documenta Math. 9 (2004): 1-27.

3. D. R. Grayson and M. E. Stillman, Macaulay 2, a software system for research in algebraic geometry, 2002. Available at http: / / www. math. uiuc. edu/Macaulay2/.

4. M. Joswig, "Tropical Halfspaces", arXiv: math.CO/0312068, 2003.

5. Maple. Available at http: / /maplesoft. com.

6. E. Miller and B. Sturmfels, Combinatorial Commutative Algebra, Graduate Texts in Mathematics, Springer, 2004.

7. R. A. Milowski, Computing Irredundant Irreducible Decompositions of Large Scale Monomial Ideals, ISSAC 2004, Santanders, Spain, 2004. Software available at http://milowski. org/software.html.

8. K. Polthier, JavaView, a 3D geometry viewer and a mathematical visualization software. Available at http: / / www.javaview.de.

9. J. Richter-Gebert, B. Sturmfels, and T. Theobald, "First Steps in Tropical Geometry", Idempotent Mathematics and Mathematical Physics, Proceedings Vienna 2003, American Mathematical Society, 2004.

10. A. Schrijver, Theory of Linear and Integer Programming, Wiley, 1986.

11. B. Sturmfels, Gröbner Bases and Convex Polytopes, University Lecture Series 8, Providence: AMS, 1996. 